\section{Trends in physician supply}

7 he national physician pool is aging but marginally larger than it was 5 years ago. Canadians are also increasingly more likely to be treated by a female physician, while it's more likely a graduate of a Canadian medical school will remain within Canada or eventually return home to hang up a shingle after a stint abroad.

Those are among trends evident in the Canadian Institute for Health Information's latest annual report on the supply, migration and distribution of physicians in Canada (2005).

The CIHI report indicates the number of physicians in Canada grew by $5.3 \%$ between $200 \mathrm{I}$ and 2005, (see Table I), although their average age increased to 48.9 years from 47.6 . Female physicians constituted $32.5 \%$ of the workforce, as compared to $30.2 \%$.

The national average of physicians per 100 ooo population is I9o, with Nova Scotia having the most at 218 , followed by Quebec 215, Yukon 205, British Columbia 199, Newfoundland \& Labrador 193, Alberta I88, Manitoba I79, Ontario I76, New Brunswick 172, Saskatchewan I56, P.E.I. 144 and N.W.T. 103. Nunavut brings up the rear with a scant 46 .

The statistics also indicate Ontario is the only jurisdiction in the country with more specialists per capita than family practitioners. In fact, Ontario is only saved by a pair of territories from being the most difficult place in the country to find a family doctor. On average, there are 98 family physicians per 100 ooo Canadians, led by Yukon I83, Nova Scotia II8, British Columbia III, Quebec I09, Alberta 102, New Brunswick 102, Newfoundland \& Labrador 99, Manitoba 94, Saskatchewan 89, PEI 89, Ontario 85 , N.W.T. $7 \mathrm{I}$ and Nunavut 43.

There are 92 specialists per 100000 Canadians, led by Quebec ro6, Nova Scotia 10o, Newfoundland \& Labrador 94, Ontario 92, British Columbia 88, Alberta 86, Manitoba 86, New Brunswick 70 , Saskatchewan 67, P.E.I. 55, NW.T. 33, Yukon 22, and Nunavut 3.- Wayne Kondro, CMAJ
Table 1: Supply, migration and distribution of Canadian physicians

\begin{tabular}{|c|c|c|c|}
\hline Variable & $\begin{array}{c}2001 \\
n=58546\end{array}$ & $\begin{array}{c}2003 \\
n=59454\end{array}$ & $\begin{array}{c}2005 \\
n=61622\end{array}$ \\
\hline No. of physicians & 58546 & 59454 & 61622 \\
\hline Family medicine & 29627 & 30662 & 31633 \\
\hline Specialist & 28919 & 28792 & 29989 \\
\hline $\begin{array}{l}\text { No. of physicians } \\
\text { population }\end{array}$ & 188 & 187 & 190 \\
\hline Family medicine & 95 & 96 & 98 \\
\hline Specialist & 93 & 91 & 92 \\
\hline \multicolumn{4}{|l|}{ Age, yr, mean } \\
\hline Family medicine & 46.4 & 47.2 & 48.0 \\
\hline Specialist & 48.9 & 49.5 & 49.9 \\
\hline \multicolumn{4}{|l|}{ Sex, male } \\
\hline Family medicine & 19217 & 19568 & 19693 \\
\hline Specialist & 21658 & 21184 & 21682 \\
\hline \multicolumn{4}{|l|}{ Sex, female } \\
\hline Family medicine & 10387 & 11000 & 11698 \\
\hline Specialist & 7260 & 7602 & 8263 \\
\hline \multicolumn{4}{|l|}{ Specialty } \\
\hline Family medicine & 29627 & 30662 & 31633 \\
\hline \multicolumn{4}{|l|}{ Medical specialist } \\
\hline Clinical & 19656 & 19758 & 20653 \\
\hline Laboratory & 1443 & 1412 & 1436 \\
\hline Surgical specialist & 7769 & 7584 & 7866 \\
\hline Medical scientist & 51 & 38 & 34 \\
\hline \multicolumn{4}{|c|}{ No. of years since graduation } \\
\hline$<6$ & 3354 & 2746 & 2560 \\
\hline $6-10$ & 7691 & 7425 & 7447 \\
\hline $11-25$ & 26786 & 26873 & 26641 \\
\hline $26-30$ & 7374 & 7935 & 8599 \\
\hline $31-35$ & 5421 & 6170 & 6805 \\
\hline$\geq 36$ & 7913 & 8304 & 9456 \\
\hline \multicolumn{4}{|c|}{ Canadian medical school graduate } \\
\hline Family medicine & 22888 & 23398 & 23988 \\
\hline Specialist & 22130 & 22436 & 23502 \\
\hline \multicolumn{4}{|c|}{ Foreign medical school graduate } \\
\hline Family medicine & 6622 & 7025 & 7264 \\
\hline Specialist & 6786 & 6353 & 6451 \\
\hline \multicolumn{4}{|c|}{$\begin{array}{l}\text { Net migration between } \\
\text { jurisdictions }\end{array}$} \\
\hline Family medicine & 417 & 380 & 372 \\
\hline Specialist & 496 & 310 & 262 \\
\hline \multicolumn{4}{|l|}{ Moved abroad } \\
\hline Family medicine & 165 & 106 & 87 \\
\hline Specialist & 390 & 189 & 99 \\
\hline \multicolumn{4}{|c|}{ Returned from abroad } \\
\hline Family medicine & 138 & 100 & 89 \\
\hline Specialist & 196 & 140 & 158 \\
\hline
\end{tabular}

Source: Canadian Institute for Health Information. 